\title{
EL USO DE LAS TIC EN LA
} FORMACIÓN EN SISTEMAS DE MEDICIÓN INTELIGENTE EN LAS INSTALACIONES DE CONSTRUCCIÓN

\section{THE USE OF ICT FOR TRAINING IN SMART METERING TECHNOLOGIES FOR CONSTRUCTION SITE MANAGERS}

Francisco Javier Cárcel Carrasco ${ }^{1}$

Elisa Peñalvo López ${ }^{2}$

1. Universitat Politècnica de València. Email: fracarc1@csa.upv.es

2. Universitat Politècnica de València. Email: elpealpe@upvnet.upv.es 


\section{RESUMEN}

El uso de las TIC para formación y en concreto para su uso en cursos masivos (MOOC), está en constante crecimiento. En este artículo, se presenta las características básicas de un proyecto de formación europeo (COSMET) para la capacitación en tecnologías para la medición inteligente de instalaciones en la edificación, donde una de sus partes fundamentales es la generación de cursos MOOC para jefes de obra y organismos de formación para profesionales, que será utilizado en toda Europa.

\section{ABSTRACT}

The use of ICT for education and specifically for use in mass courses (MOOC), is constantly growing. This article presents the basic characteristics of an European training project (COSMET) for training in smart metering technologies for construction site managers in the building, where one of its key parts is the generation of MOOC courses for site managers and VET, which will be used throughout Europe.

\section{PALABRAS CLAVE}

Medición inteligente; Formación; Jefes de obra; Cursos MOOC

\section{KEY WORDS}

Smart metering technologies; Training; Construction site managers; Courses MOOC 


\section{INTRODUCCIÓN}

Una de las tendencias de la actual sociedad de la información es la necesidad de formación permanente en torno a una realidad en constante evolución. Los MOOC suponen un recurso muy interesante para satisfacer esa creciente demanda de formación en todo el mundo de un público cada vez más numeroso y global.

Aprendizaje y formación son piezas claves para actualizar los conocimientos y habilidades a nuevos avances tecnológicos, tales como sistemas inteligentes de medición en los edificios (electricidad, gas, agua, etc.) para jefes de obra y supervisores de construcción y otros profesionales que actúan en la construcción. Este artículo muestra las principales características, etapas y expectativas de un proyecto de formación europea (web 1) comenzada en el año 2015 hasta 2017 (proyecto COSMET dentro de ERASMUS + Programme financiado por la comisión europea), con el fin de estudiar las necesidades de formación de los administradores del edificio en relación con las medidas inteligentes y diseño de cursos para la actualización de conocimientos de estos profesionales.

La prioridad del proyecto es lograr resultados para mejorar la relevancia de las asociaciones para formación de estos profesionales y jefes de obra por la modernización de la supervisión de la construcción y habilidades mediante una formación integral. Para atender esta prioridad, el proyecto forma una alianza estratégica que reúne a centros de formación para profesionales, asociaciones de colectivos y universidades. Después de esta prioridad, el proyecto desarrollará un curso integral de medición inteligente (medición de agua, gas, calor y energía), y capacitar a los administradores de asociaciones profesionales con las habilidades apropiadas para fortalecer su empleabilidad en el mercado de la UE, y abordar sus habilidades digitales y medio-ambientales que en la actualidad.

El proyecto también apoya la prioridad para producir recursos educativos abiertos originales en los idiomas de la Asociación para mejorar la absorción de las TIC en formación, promoviendo el acceso fácil y gratuito a disposición de los centros de formación, permitiendo capacitación y el cumplimiento de los objetivos de la apertura a la iniciativa de educación. 


\section{CARACTERÍSTICAS DE LOS CURSOS MOOC}

Los "MOOC" "Massive Open Online Course" son una modalidad formativa que se oferta desde un espacio en Internet y que contempla una inscripción abierta y, por tanto, un acceso de un elevado número de participantes.

Con el fenómeno de los $\mathrm{MOOC}$, estamos encontrando una doble disyuntiva: los que creen que puede ser una tecnología, o mejor dicho, una aplicación educativa tecnológica, que facilite y garantice que una formación de calidad pueda llegar a todas las personas independientemente de su lugar y clase social, y los que creen que este nuevo medio no es nada nuevo y simplemente supone un nuevo modelo de negocio para las Universidades y las Instituciones, pero que su calidad deja mucho que desear (Zapata, 2013; Vázquez et al., 2013), y suponen un retroceso en la aplicación educativa de las Tecnologías de la Información y la Comunicación (TIC) (Cabero et al., 2014).

La idea de que las tecnologías emergentes en educación pasan por ciclos de sobreexpectación y la evidente analogía con la realidad de las pedagogías emergentes, nos remite a la Curva de Gartner (figura 1) (Linden y Fenn, 2003) como modelo explicativo comúnmente aceptado, aunque escasamente contrastado (Steinert y Leifer, 2010). Así, tal y como se plantea en la Curva de Gartner, en las pedagogías emergentes "la primera parte de la curva de sobreexpectación está impulsada por una expectativas infundadas -creadas principalmente por los medios, que especulan sobre las perspectivas de la tecnología. La segunda parte de la curva está impulsada por mejoras en el rendimiento y el crecimiento de la adopción" (Hernández et al., 2014).

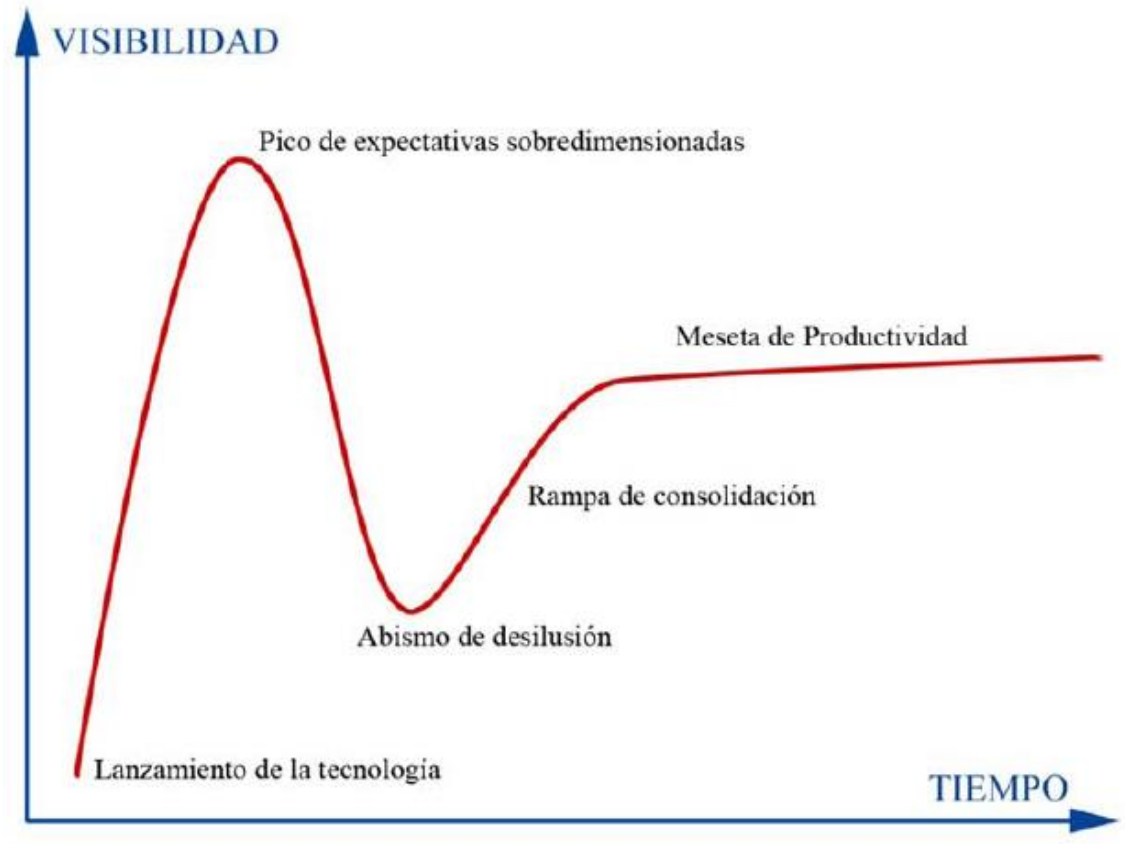

Figura 1. Ciclo de sobreexpectación de las tecnologías según Gartner (Adaptado por Cabero et al., 2014). 
Con el uso de las TICs, los MOOC tienen una característica muy flexible y engloba variantes muy dispares en función de parámetros adicionales: inscripción gratuita o de pago, presencia o ausencia de tutores, rol del profesorado como animador o experto, fechas concretas de realización o flexibilidad en las mismas, contenidos cerrados o abiertos, etc.

De una manera básica, las características mínimas que debe tener un MOOC son (Web 2):

- Formato de curso. Representa una unidad completa de formación con un itinerario que contiene elementos informativos multimedia, tareas a realizar y pruebas que acrediten el conocimiento adquirido por el alumnado.

- Masivo. Está pensado para cualquier persona del mundo global y el número de matriculados en principio no está limitado siendo muy superior a un curso presencial ordinario.

- Inscripción abierta. Cualquier usuario de Internet puede acceder a los contenidos del curso.

- En línea. Es un recurso pensado para la formación a distancia donde Internet es el único medio de comunicación.

- Duración reducida. Está previsto realizarse en un cierto periodo de tiempo de varias semanas.

Pero también tiene una serie de inconvenientes que es necesario mencionar (Web 2):

- Abandono. La mayoría del alumnado que se inscribe en un MOOC no suele terminarlo.

- Requisitos previos. La inscripción abierta favorece en ocasiones el acceso de alumnado que no dispone de los conocimientos necesarios para superar el curso con éxito.

- Tiempo de duración. Se debe tender a cursos de tiempo reducido. Si el tiempo es muy elevado, la tasa de abandono aumenta.

- Diversidad. Hay que tener en cuenta la situación inicial del alumno: conocimientos previos, necesidades formativas, etc.

- Calidad. Existen algunos cursos abiertos que no gozan de la calidad de diseño necesaria para que el alumnado invierta su tiempo en realizarlos.

- Gratuidad. Los MOOC surgieron en un principio como iniciativas gratuitas, aunque han surgido diferentes iniciativas de pago para su financiación.

- Tutoría. En ocasiones, la gratuidad o el carácter masivo de una iniciativa MOOC no permite garantizar la presencia de expertos que puedan animar el curso, moderar los foros, resolver las dudas y evaluar las tareas enviadas por los participantes. 
- Accesibilidad. Los MOOC suelen estar repletos de vídeos por lo que demandan una conexión a Internet de última generación y, por otro lado, suelen estar en inglés lo cual deja fuera a aquellas personas que no dominan este idioma.

- Acreditación. También suelen existir problemas para que una institución de reconocido prestigio certifique la superación del curso. No solo porque entra en competencia con la propia enseñanza reglada y/o presencial que puede impartir esa misma entidad, sino también porque puede no resultar muy riguroso a falta de una prueba presencial que certifique la identidad real del participante.

Estos inconvenientes en su mayor parte pueden resolverse en un corto o medio plazo. En el futuro, el auge de los MOOC dependerá de cómo se solucionen estas deficiencias. Así, por ejemplo, para evaluar y corregir las tareas a un elevado número de alumnos. Como variante organizativa se puede plantear que una actividad del alumno/a sea evaluada por dos o más alumnos/as elegidos al azar situados más adelante en el curso utilizando una rúbrica como una tarea propia del mismo.

\section{TIPOS DE MOOC}

Los tipos generales de MOOC, pueden tener diferentes conceptos. En múltiples referencias se maneja el concepto C-MOOC para referirse a los cursos orientados al aprendizaje conectivo. Este término surge en oposición al concepto X-MOOC referido a los cursos alojados en plataformas más rígidas como pueden edX y otras de índole similar.

A continuación, en la tabla, 1 se exponen algunas ideas sobre el uso educativo de los MOOC en el contexto de un aula.

\begin{tabular}{|c|c|}
\hline Contenidos abiertos & $\begin{array}{l}\text { El uso de software libre y contenidos abiertos predispone } \\
\text { también hacia el aprovechamiento de los MOOC disponibles. }\end{array}$ \\
\hline Unidades de aprendizaje & $\begin{array}{l}\text { Un MOOC pone de manifiesto la importancia del curso como } \\
\text { unidad básica de formación. La tendencia poner en valor } \\
\text { propuestas de aprendizaje más complejas que faciliten al } \\
\text { profesorado las tareas de preparación y reutilización. }\end{array}$ \\
\hline Variedad & $\begin{array}{l}\text { Disponen de páginas de información, vídeos, cuestionarios y } \\
\text { foros. Convendría dotar de variedad a los cursos que se ofrecen. }\end{array}$ \\
\hline Vídeos & $\begin{array}{l}\text { Los } \mathrm{MOOC} \text { pueden estar repletos de vídeos. Conviene moderar e } \\
\text { uso de este recurso en el diseño de un curso porque la conexión } \\
\text { a Internet resulta deficitaria todavía en muchos centros y } \\
\text { hogares. }\end{array}$ \\
\hline Autofor & $\begin{array}{l}\text { Al orientar al alumnado hacia la realización de cursos abiertos en } \\
\text { línea con cierto apoyo tutorial desde el aula, resulta evidente } \\
\text { que se le está familiarizando con su futuro formativo en la red. }\end{array}$ \\
\hline La barrera idiomática & $\begin{array}{l}\text { La mayoría de } \mathrm{MOOC} \text { están en inglés. De hecho se suele } \\
\text { recomendar la creación del curso en este idioma para asegurar } \\
\text { su carácter masivo. }\end{array}$ \\
\hline Plataformas & $\begin{array}{l}\text { Existen multitud de plataformas para la creación de MOOC. Pero } \\
\text { un } \mathrm{MOOC} \text { no es una estructura de curso sino una filosofía de }\end{array}$ \\
\hline
\end{tabular}




\begin{tabular}{|l|l|}
\hline Sostenibilidad & $\begin{array}{l}\text { diseño. } \\
\text { El diseño de MOOC con cierto grado de calidad no resulta } \\
\text { gratuito. }\end{array}$ \\
\hline $\begin{array}{l}\text { Colaboración } \\
\text { institucional }\end{array}$ & $\begin{array}{l}\text { educativas) pueden encontrar puntos comunes en el diseño de } \\
\text { MOOC de calidad en torno a temáticas transversales. }\end{array}$ \\
\hline Evaluación seria & $\begin{array}{l}\text {. El uso educativo de MOOC en el ámbito educativo reglado se } \\
\text { puede complementar con una evaluación presencial como factor } \\
\text { de corrección y ajuste al seguimiento y superación de sus } \\
\text { contenidos }\end{array}$ \\
\hline
\end{tabular}

Tabla 1. Uso educativo de los MOOC. Fuente: Adaptado a partir de Web 2.

Uno de los problemas asociados a la utilización de los $\mathrm{MOOC}$ es el de la identificación de los estudiantes, aunque es una preocupación que es menor que en otro tipos de acciones de formación virtual, ya que una de sus características es que las personas suelen inscribirse de forma voluntaria (Becerra, 2013). 


\section{EL OBJETIVO DEL PROYECTO COSMET}

Según Eurostat, existen en la UE_27, aproximadamente 882.000 empresas que operan en la construcción del sector edificios, empleando a 3,9 millones personas, de donde existen aproximadamente 260.000 jefes y gestores de obra en la construcción. Estos técnicos son responsables de la construcción de un edificio, supervisar las operaciones en el día a día y asegurando que se trabaja de forma segura y con los estándares de la calidad adecuada.

Más allá de la instalación de contadores inteligentes que se llevan a cabo en la mayoría de la UE por instaladores cualificados, las infraestructuras de medición inteligentes están interconectadas a otras características del edificio. Los jefes de obra necesitan actualizar su formación para adquirir conocimientos adicionales para responder a necesidades de la construcción moderna que involucran soluciones integrales para el control de energía, gas, calor, etc.

Hay una necesidad de formación de estos técnicos y jefes de obra, viendo la necesidad de generar cursos sobre medición inteligente en las instalaciones de los edificios con respecto: a) principios generales de medición inteligentes y desafíos, b) técnicas y sistemas de organización para optimizar la interconexión de las infraestructuras. Para abordar esta necesidad, es necesario desarrollar recursos educativos abiertos que asegurar el fácil y libre acceso a material educativo en línea, promoción de la autoformación y autoevaluación en el propio tiempo, lugar y ritmo.

El proyecto forma una alianza estratégica para modernizar los centros de formación para estos profesionales con el objetivo de:

Objetivo 1: Desarrollar resultados de aprendizaje y cursos sobre medición inteligente de electricidad, gas, calor y agua, para mejorar la disposición pertinente de los jefes de obra.

Objetivo 2: Introducir métodos de entrenamiento modernos en forma de enseñanza y evaluación de materiales, y curso en línea abierto masivo (MOOC).

Objetivo 3: Facilitar el reconocimiento mutuo de los resultados de aprendizaje desarrollados en toda UE.

Los grupos objetivo del proyecto incluyen:

- Estudiantes con la intención de trabajar como jefes o directores de obra.

- Gestores técnicos que trabajan en el sector de la construcción en Europa.

- Los proveedores de formación para los administradores de sitios de construcción.

- Las partes interesadas y asociaciones en el sector de la construcción. 


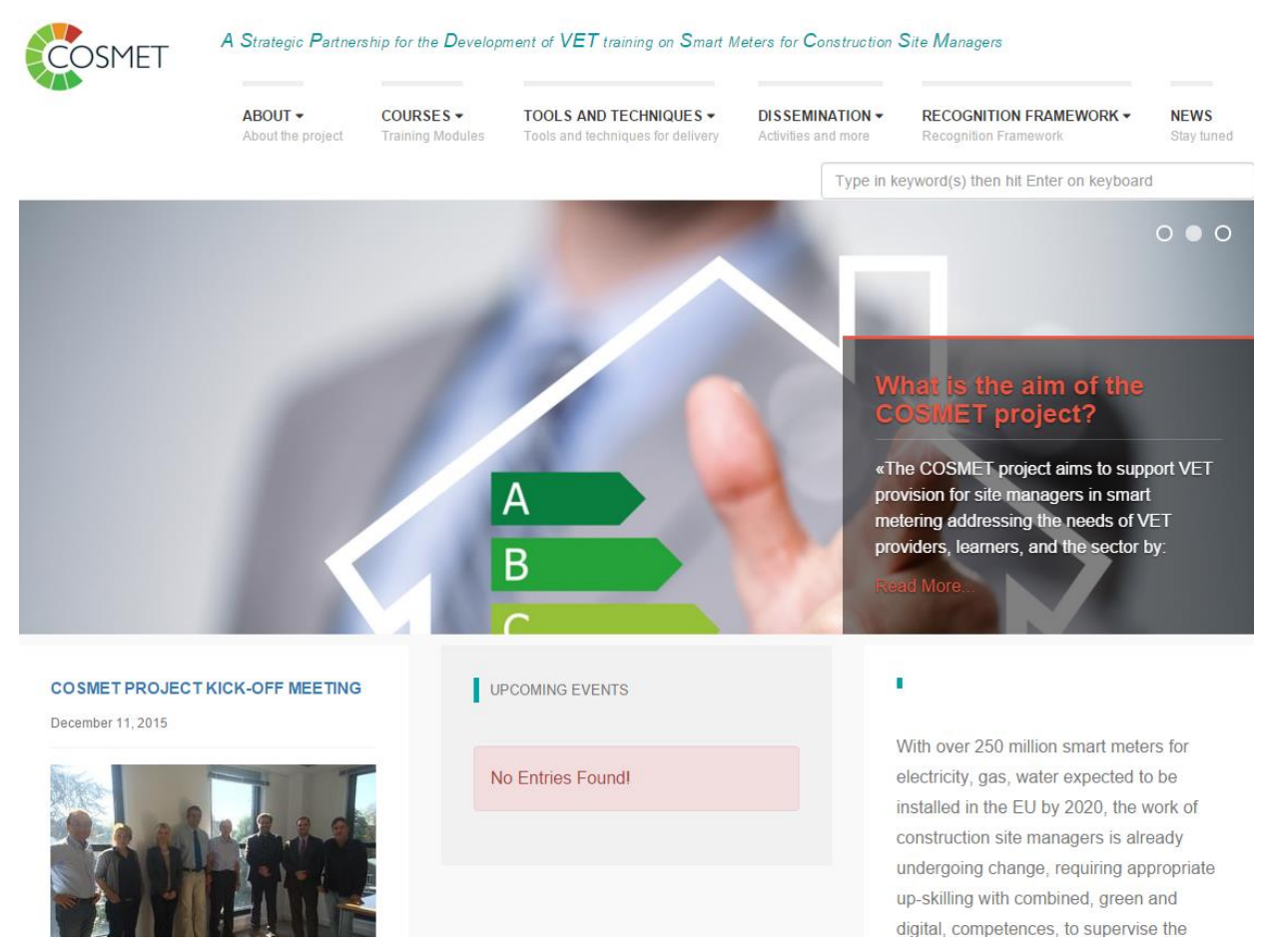

Figura 2. Web realizada para el proyecto Cosmet. Fuente: Web 3.

Hay seis socios que forman parte de este proyecto de formación usando las TIC. Cinco centros formados por asociaciones de profesionales o de formación y una Universidad Europea. Los socios que integran esta asociación son:

Summit Skills (http://www.summitskills.org.uk/) es la organización de estandarización para el sector de la construcción y servicios de Ingeniería en el Reino Unido.

PROMEA. (www.promea.gr) La Sociedad Helénica para la promoción de la investigación y las metodologías de desarrollo, tiene como objetivo la promoción y mejora de la sociedad del conocimiento en Grecia y Europa.

PSMB. La Asociación polaca de gerentes del edificio (http://www.psmb.pl/mbaic-2/) se formó en colaboración con la Asociaciones de construcción europeas y expertos de la construcción con el fin de educar y certificar a personal de la industria de la construcción polaca.

La Universitat Politécnica de Valencia (http://www.upv.es/) es una institución académica pública que se dedica a la investigación y enseñanza en el campo de las tecnologías de construcción. Mantiene fuertes lazos con su entorno social y una fuerte presencia en el exterior, proporciona a los estudiantes educación integral con conocimientos técnicos.

BFW NRW, el Instituto de formación profesional de la industria de la construcción en NorteWestfalia (http://www.berufsbildung-bau.de/englishinformation/) es un centro de formación especializado en formación profesional y educación continua como una institución sin fines de lucro para la formación inicial y continua de los trabajadores de la industria de la construcción. 
VSRC Centro de formación de constructores Vilna

(http://www.vsrc.lt/joomla/english/about-us.html) es una institución profesional de formación profesional y capacitación. El centro ofrece una amplia gama de cursos y excelentes instalaciones en área de la construcción.

Innovación del proyecto:

El consorcio del proyecto producirá el plan de estudios disponible en el formato de un MOOC para apoyar el aprendizaje informal y personal, como un medio para llegar mediante las nuevas tecnologías de las comunicaciones, a sistemas de capacitación y formación continua.

El proyecto adoptará un aprendizaje con un enfoque en el diseño del currículo para conectar el programa de formación desarrollado con las herramientas de referencia Europeas. Este enfoque contribuirá a la promoción de un lenguaje común en las habilidades desarrolladas. 


\section{RESULTADOS E IMPACTO DEL PROYECTO COSMET}

La lista siguiente proporciona un resumen de los resultados intelectuales y eventos del proyecto COSMET, basado en el aprendizaje con el uso de las TIC:

1. Evidencias en los resultados del aprendizaje para el suministro de formación en habilidades de medición inteligentes integrales para jefes y directores de obra (01).

2. Unidades de aprendizaje formales para el curso COSMET y directrices de integración de entidades de formación continua de profesionales (O2).

3. Recursos pedagógicos y herramientas de evaluación para habilidades de medición inteligentes para directores de obra, así como recursos de enseñanza abiertos (O3).

4. COSMET MOOC en habilidades de medición inteligentes integrales para administradores del sitio (O4).

5. Marco para el reconocimiento de los resultados hacia una medición inteligente para la calificación de jefes de obra (O5).

6. Dos talleres de demostración en Lituania y Alemania para promover resultados COSMET y validar materiales de aprendizaje COSMET (E1-E2).

7. Cuatro días de información nacional en el Reino Unido, España, Grecia y Polonia para compartir y difundir los resultados de COSMET (E3-E6).

El proyecto pretende un impacto significativo en los alumnos, y potenciar habilidades y competencias clave necesarias en el sector:

-Asociaciones de profesionales en el sector de la construcción: Mejorará su eficacia y su productividad, satisfaciendo las demandas de trabajos actuales y emergentes en cuanto a servicios y tecnologías de medición inteligentes.

-Jefes de obra sénior: Mejorará su sostenibilidad de empleo reduciendo sus carencias de habilidades resultantes de la penetración con los avances digitales y tecnológicos en el sector de la construcción.

-Formación continua para estudiantes y solicitantes de empleo que deseen trabajar como gestores de obra de la construcción: se posicionan mejor en el mercado de trabajo y aumentar su empleabilidad.

El consorcio COSMET espera que el proyecto también tendrá impacto en los interesados en el sector más allá del alcance directo del proyecto:

-Interlocutores, representantes del sector, entidades de formación nacionales y las autoridades europeas podrán aprovechar el desarrollo de un marco común para la certificación y acreditación de las habilidades en medición inteligente de los recursos de los edificios con el fin de aumentar su eficiencia y ahorro energético. 
-Constructoras y grupos de interés sectoriales podrán aprovechar la coincidencia esperada de habilidad oferta y demanda en el largo plazo (después del proyecto), que dará como resultado mayor productividad y competitividad, con beneficios económicos.

- Materiales de aprendizaje se desarrollará en forma modular (resultados de aprendizaje y unidades de aprendizaje).

El impacto esperado del proyecto a nivel local, los niveles regionales, nacionales, europeos o internacionales contribuirá a la consecución del objetivo 2020 en educación y empleo en la Comisión Europea, buscando aumentar el número de ciudadanos de la UE que participan en la educación post-secundaria. 


\section{MEDIDA DEL IMPACTO DEL PROYECTO COSMET}

El impacto se medirá tanto cuantitativa como cualitativamente y se monitorearán durante todo el proyecto, lo que permite acciones correctivas si son necesarias. Esta evaluación continua se expresará en informes de análisis de impacto anuales.

La evaluación cualitativa se centrará sobre todo en los posibles beneficios a largo plazo para estudiantes y el sector de formación continua en las actividades de construcción. Con la oportunidad de diversos eventos, se observarán las tendencias en los participantes empleando cuestionarios y entrevistas para recopilar evaluaciones de resultados y para identificar factores externos que influyen en la absorción de los resultados del aprendizaje del proyecto COSMET. Además, se tiene como objetivo evaluar el impacto del proyecto a través de la campaña de correo electrónico para agencias de cualificaciones nacionales y las autoridades para reunir información sobre el plan de acción y colaboración de COSMET, durante los últimos meses y después del proyecto.

Para evaluar el impacto, los socios integrantes solicitarán continuamente aportar pruebas antes, durante y después de la implementación de cada actividad, tales como: a) las listas de participantes a eventos, b) los registros de acceso y uso de herramientas de aprendizaje en línea y canales de difusión (por ejemplo, actividad de los medios de comunicación social), c) registros de impacto de herramientas de evaluación, d) comunicación (es decir, cartas a los proveedores de formación de asociaciones y estudiantes), las partes interesadas y los responsables políticos, etc.

Sobre la base de esta evidencia y en yuxtaposición con los indicadores de rendimiento clave antes mencionadas, el análisis evaluará cómo de influyente es el impacto del proyecto. 


\section{CONCLUSIONES}

El proyecto COSMET está diseñado para ser sostenible y útil a las asociaciones que imparten formación continua a profesionales de la construcción en la EU-28, actores sectoriales y autoridades públicas involucrados en la formulación de políticas para: a) formación y b) la promoción de la movilidad y la transparencia de cualificaciones.

Con este fin, los resultados del proyecto serán libremente accesibles por el público, bajo la licencia Creative Commons 4.0 para permitir su libre uso, distribución y modificación de materiales. Esta licencia sólo requiere referencia al creador, que se define como la asociación estratégica de COSMET bajo el programa Erasmus +. Específicamente:

a) Los recursos de aprendizaje serán desarrollados y empaquetados como recursos educativos abiertos sin restricciones al uso, algo conveniente para la integración o intercambio en plataformas como la plataforma electrónica para el aprendizaje de adultos en Europa (EPALE), patrocinado por el programa Erasmus + y sistemas de gestión de aprendizaje de los proveedores de formación.

b) Los resultados de aprendizaje y unidades de aprendizaje por módulos que se desarrollarán seguirán la estructura semántica subyacente para garantizar la interoperabilidad. Será descargable desde la página web del proyecto en formato digital y los grupos pueden mejorar con datos actualizados de la industria.

c) Todos los informes del proyecto y los medios de difusión será descargables a través de la página web del proyecto y los medios de comunicación social.

d) La Asociación aplicará para alojar el MOOC en la plataforma patrocinada por la Comisión Europea "iversity" (DE), que utiliza la infraestructura de código, o en otras plataformas europeas en abierto.

Los MOOC pueden revolucionar la forma de aprender en red, ya que representan un formato libre, abierto, masivo y gratuito de conseguir formación sobre temas de interés. La experiencia acumulada por el alumnado participante contribuye a prepararles o mejorar profesionalmente, teniendo en cuenta que la formación permanente y en línea utilizando las TIC resultará imprescindible para mejorar la competitividad y productividad en un futuro. 


\section{AGRADECIMIENTOS}

Este trabajo ha sido realizado dentro del marco del proyecto COSMET "Training in Smart Meters for Construction Site Managers" financiado por la Comisión Europea dentro de la Key Action 2: Strategic Partnerships in VET, Numero de Referencia 2015-1-UK01-KA202-013406. 


\section{REFERENCIAS BIBLIOGRÁFICAS}

Becerra, O. (2013). The One Laptop Per Child Correlation With Massive Open Online Courses. Recuperado de: <http://edutechdebate.org/massive-open-online-courses/the-onelaptop-per-child-corollation-with-massive-open-online-courses/>

Cabero Almenara, J., Llorente Cejudo, M. D. C., y Vázquez Martínez, A. I. (2014). Las tipologías de MOOC: Su diseño e implicaciones educativas. Revista profesorado, 18 (1).

CanalTic. (2016). <http://canaltic.com/blog/?p=1772/>

Hernández Ortega, J., Prennesi Fruscio, M., Sobrino López, D., y Vázques Gutiérrez, A. (2014). Tendencias emergentes en Educación con TIC.

Linden, A. y Fenn, J. (2003). Understanding Gartner's Hype Cycles. Strategic Analysis Report R-20-1971. Gartner Research.

Steinert, M. y Leifer, L. (2010). Scrutinizing Gartner's hype cycle approach. Technology management for global economic growth (PICMET), Proceedings of PICMET'10, pp. 113. Recuperado de: <http://ieeexplore.ieee.org/xpls/abs all.jsp?arnumber=560344/>

The EU programme for education, training, youth and sport. (2016) <http://ec.europa.eu/programmes/erasmus-plus/index es.htm/>

The web of Cosmet project. (2016). <http://www.etaew.net/cosmet/>

Vázquez, E., López, E. y Sarasola, J.L. (2013). La expansión del conocimiento abierto: los MOOC. Barcelona: Octaedro.

Zapata, M. (2013). MOOCs, una visión crítica y una alternativa complementaria: La individualización del aprendizaje y de la ayuda pedagógica. Campus Virtuales, II (1), pp 20-38. 